\title{
Fabrication and Characterization of InGaN Nano-scale Dots for Blue and Green LED Applications
}

\author{
K.S. Kim, C.-H. Hong, W.-H. Lee, C.S. Kim, O.H. Cha, G.M. Yang, E.-K. Suh, K.Y. \\ Lim, H.J. Lee, H.K. Cho ${ }^{1}$, J.Y. Lee ${ }^{1}$, and J.M. Seo ${ }^{2}$. \\ Department of Semiconductor Science \& Technology and Semiconductor Physics \\ Research Center, Chonbuk National University, Chonju 561-756, Korea \\ ${ }^{1}$ Department of Materials Science and Engineering, KAIST, 373-1 Kusong-Dong, \\ Yusong-gu, Taejon 305-701, Korea \\ ${ }^{2}$ Department of Science and Technology, Chonbuk National University, Chonju 561-756, \\ Korea
}

\begin{abstract}
Thin layers of InGaN were grown by metalorganic chemical vapor deposition and characterized with atomic force microscopy and high-resolution transmission electron microscopy. InGaN deposited on $\mathrm{GaN}$ exhibits a Stranski-Krastanov growth mode, including 2D wetting layer and 3D self-assembled quantum dots. Besides, we observed that the formed InGaN nano-scale dots have a trapezoidal shape with a $\{1-102\}$ facet with respect to (0002) surface. Visible spectral range from UV to green was easily obtained by changing InGaN quantum well thickness up to $2.3 \mathrm{~nm}$.
\end{abstract}

\section{INTRODUCTION}

The $\operatorname{In}_{\mathrm{x}} \mathrm{Ga}_{1-\mathrm{x}} \mathrm{N}$ alloys have been used as an active layer material of InGaN based blue and green light emitting diodes (LEDs) and laser diodes (LDs) because they cover a wide band-gap ranging from 1.9 to $3.4 \mathrm{eV}$. Especially, InGaN/GaN quantum well (QW) is center of interest on account of their huge applications in high brightness LED, [1] cw blue LD, [2] and optoelectronic devices. Nevertheless, the emission and growth mechanisms of InGaN/GaN QW structure is still equivocal and debating.

In recent, a few research groups have suggested that the optical emission mechanism in InGaN/GaN QW should be due to InGaN quantum dot (QD). [3] Another groups, on the other hand, asserted that the emission from the InGaN/GaN QW stems from piezo-electric effects, [4] quantum confined Stark effects [5] or potential fluctuation of InGaN QW.

In this work, we investigate the time dependent 2D to 3D mophological evolution of InGaN thin films grown by metalorganic chemical vapor deposition (MOCVD) through atomic force microscopy (AFM), high resolution transmission electron microscopy (HRTEM) measurements. Besides, their related optical emission characteristics in InGaN/GaN SQW using photoluminescence (PL) measurement.

\section{EXPERIMENTS}

All InGaN/GaN QW structures used in this study were grown by low pressure horizontal MOCVD. InGaN/GaN SQW structures are consisted of a $\mathrm{GaN}$ nucleation layer with a nominal thickness of $25 \mathrm{~nm}$ grown at $560{ }^{\circ} \mathrm{C}$, a Si-doped $\mathrm{GaN}$ layer with a thickness of $1.8 \mathrm{um}$ grown at $1100{ }^{\circ} \mathrm{C}$, a low-temperature grown $\mathrm{GaN}$ with a thickness of 

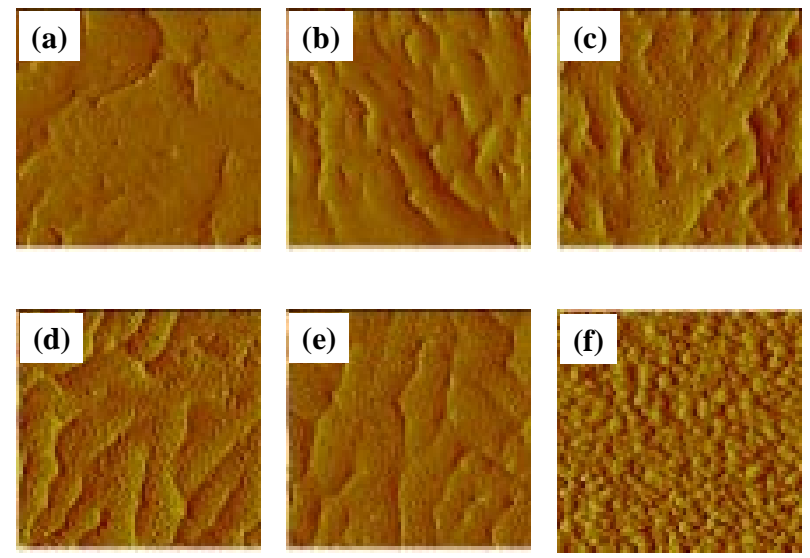

Figure 1, AFM images of InGaN films with the growth time (nominal thickness) of (a) $0 \mathrm{sec}(0 \mathrm{~nm}),($ b) $3 \mathrm{sec}(0.15 \mathrm{~nm}),(\mathrm{c})$ $12 \mathrm{sec}(0.57 \mathrm{~nm}),(d) 24 \mathrm{sec}(1.15 \mathrm{~nm}),(e) 48 \mathrm{sec}(2.3 \mathrm{~nm})$, and (f) $3600 \mathrm{sec}(400 \mathrm{~nm})$. Scan areas are $4 \times 4 \mathrm{um}^{2}$.

$5 \mathrm{~nm}, \mathrm{InGaN}$ SQW applying different growth conditions, and a $30 \mathrm{~nm}$-thick GaN capping layer grown at $1100{ }^{\circ} \mathrm{C}$. The precursors of $\mathrm{Ga}, \mathrm{In}, \mathrm{Si}$, and $\mathrm{N}$ are trimethylgallium, trimethylindium, silane, and ammomia, respectively.

\section{RESULTS AND DISCUSSION}

Figure 1 (a) - (f) show the AFM images of morphological evolution of InGaN thin films grown at $800{ }^{\circ} \mathrm{C}$, which are dependent on the growth times (thickness) from $0 \mathrm{sec}$ to $3600 \mathrm{sec}$. Typical surface shape for n-type GaN, including steps, terraces, and surface dislocations, is shown in Fig. 1 (a). The small surface dislocations observed are $37 \mathrm{~nm}$ in size and $0.5-1 \mathrm{~nm}$ in height. The density of surface dislocations is $4-5 \times 10^{9} \mathrm{~cm}^{-2}$, which shows good agreement with the dislocation density measured by the TEM. Many of them are lie along the step boundaries. As the nominal thickness of InGaN films increase from 0.15 to $1.15 \mathrm{~nm}$, one can clearly see that $2 \mathrm{D}$ (corresponding to wetting layer) to $3 \mathrm{D}$ (self-assembled QD) transition, which is direct evidence of StranskiKrastanov (S-K) growth mode of InGaN epilayer. For the case of QDs shown in the InGaN film with the thickness of $0.57 \mathrm{~nm}$ and $1.15 \mathrm{~nm}$, the average lateral size and height of dots are $73 \mathrm{~nm}, 2 \mathrm{~nm}$, and $78 \mathrm{~nm}, 3.7 \mathrm{~nm}$, respectively. Especially, the density $\left(4 \times 10^{9} \mathrm{~cm}^{-2}\right.$ ) of QDs shown in Fig. 1 (c) is nearly same with that of surface dislocations of low-temperature grown GaN. However, we have confirmed they have no correlation each other (see Fig. 2 (a)). If the thickness more increases, the size of the InGaN nanoscale dots is increased and the InGaN film shows polycrystalline growth mode or cluster formation.

From the x-ray photoelectron spectroscopy measurement (not shown here), we observed that In $3 \mathrm{~d}$ peaks intense with increasing the thickness of InGaN thin films. This result shows that In surface segregation is occurred during the InGaN growth[6].

Figure 2 (a) and (b) show a plan-view HRTEM and a cross sectional HRTEM (XHRTEM) image of the sample in Fig. 1 (c). The most important feature in (a) is that the nano-scale dots are formed irrespective of dislocation indicated by an arrow. Especially, 

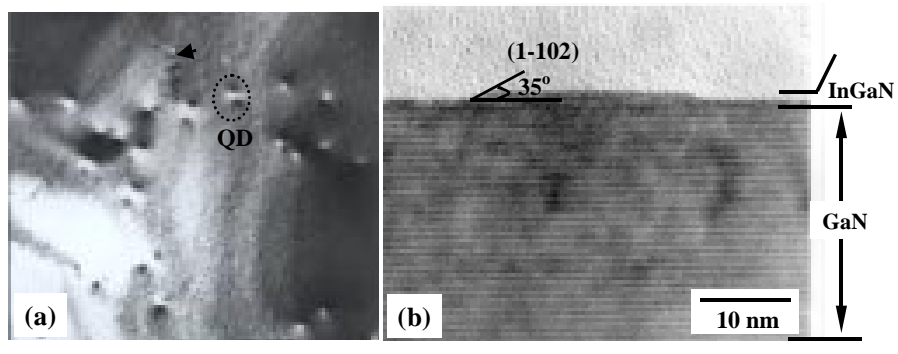

Figure 2, (a) Plan-view HRTEM weak beam image and (b) X-HRTEM image of $0.57 \mathrm{~nm}$-thick InGaN thin film. Arrow shown in (a) indicates dislocations.

knowledge of the shape of self-organized InGaN/GaN QDs is needed to constrain theoretical calculations of the process formation as well as electronic structure. For the heteroepitaxial growth suffering large lattice mismatch like InAs/GaAs and $\mathrm{Ge} / \mathrm{Si}$, several quantum dot shapes, namely conical[7], square-base pyramid[8], prism-like cluster[9], triangular shape [10] etc., have been reported. Figure (b) shows that the InGaN nano-scale dot with trapezoidal shape are formed on InGaN wetting layer without dislocations and also has $\{1-102\}$ facet planes inclined by $35^{\circ}$ with respect to the $(0002)$ surface.

Fig 3 (a) shows the room temperature PL spectra of InGaN/GaN SQW with the InGaN thickness described in Fig. 1. The FWHM values are $70-140 \mathrm{meV}$. The PL
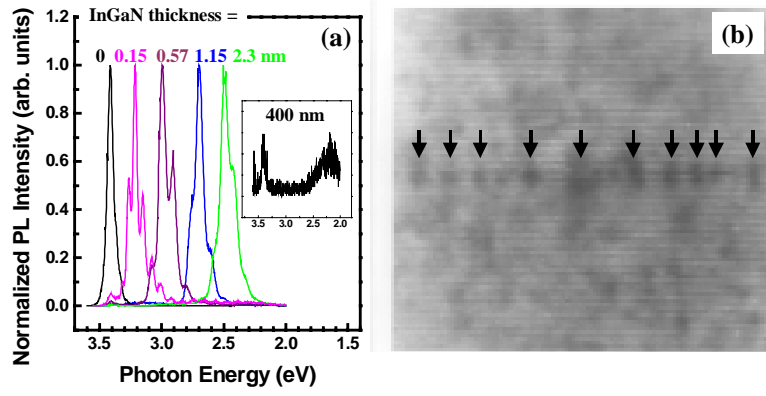

Figure 3, (a) Room temperature PL spectra of InGaN/GaN $S Q W$ with various InGaN thickness, and (b) X-HRTEM image of InGaN/GaN SQW with the InGaN QW thickness of $0.57 \mathrm{~nm}$. Arrows point out the formed InGaN nano-scale dots.

spectra were recorded using the $325 \mathrm{~nm}$ line of a He-Cd laser with an excitation power density of $2 \mathrm{~W} / \mathrm{cm}^{2}$. As we changing InGaN QW thickness from 0 to $2.3 \mathrm{~nm}$, broad area spectra covering from UV $(3.4 \mathrm{eV})$ to green $(2.45 \mathrm{eV})$ are observed. Nano-scale dots formed in InGaN SQW with the thickness of $0.57 \mathrm{~nm}$ are displayed in Fig. 3 (b), which definitely affect the emission from the InGaN SQW. Therefore, in order to explain the peculiar behavior of large red shift $(900 \mathrm{meV})$ shown in (a), we have to consider not only 


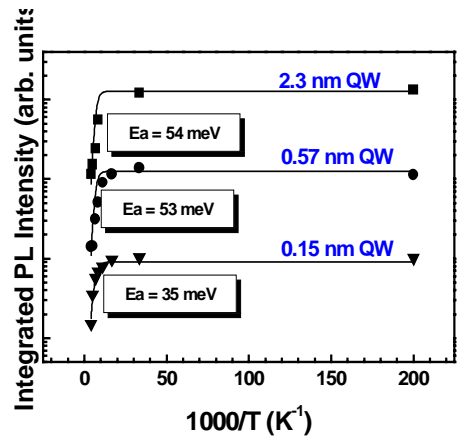

Figure 4, Arrhenius plot of the integrated PL intensity of the InGaN SQW samples with the InGaN $Q W$ thickness of 0.15 (down triangle), 0.57 (circle), and $2.3 \mathrm{~nm}$ (square), respectively

both QD confinement effect and QW thickness variation but also In surface segregation effect, piezoelectric effect etc..

Temperature dependent PL for the InGaN SQWs with the InGaN thickness of 0.15, 0.57 , and $2.3 \mathrm{~nm}$ was performed in the temperature range of $5 \mathrm{~K}$ to $300 \mathrm{~K}$. In Fig. 4, the variations of integrated PL intensities of respective sample are plotted as functions of the inverse of temperatures. The thermo-activation energy for bound excitonic emission obeys the following relation[11]:

$$
\mathrm{I}(\mathrm{T})=\mathrm{I}_{0} /\left[1+\mathrm{CT}^{3 / 2} \exp \left(-\mathrm{E}_{\mathrm{a}} / \mathrm{k}_{\mathrm{B}} \mathrm{T}\right)\right]
$$

where $\mathrm{I}(\mathrm{T})$ is the integrated PL intensity at temperature of $\mathrm{T}$ and $\mathrm{I}_{0}$ and $\mathrm{C}$ are constants. $\mathrm{k}_{\mathrm{B}}$ is Boltzmann's constant. $\mathrm{E}_{\mathrm{a}}$ is equal to the thermo-activation energy. The solid lines in Fig. 4 denote the least-square fit of the experimental data by Eq. (1). For the cases of InGaN SQW specimens with the InGaN thickness of $0.15,0.57$, and $2.3 \mathrm{~nm}$, the estimated thermo-activation energies are 35, 53, and $54 \mathrm{meV}$, respectively. This result reveals that PL emission from InGaN SQW samples embedded on InGaN 3D nano-scale dots suffer more additional quantum confinement energy of nearly $20 \mathrm{meV}$ than the case of embedded on InGaN 2D wetting layer.

\section{SUMMARY}

Thin layers of InGaN as a function of thickness were grown by MOCVD and characterized using AFM and HRTEM. InGaN thin films grown on GaN reveals a Stranski-Krastanov growth mode,. From the plan-view HRTEM measurement, we observed the creation of InGaN self-assembled QD is not related with dislocations. We have also found that the formed InGaN nano-scale dots have the trapezoidal shape with a \{1-102\} facet. Visible spectral range from UV to green was easily obtained by changing InGaN quantum well thickness up to $2.3 \mathrm{~nm}$. From the integrated PL intensity analysis, emission from InGaN SQW samples embedded on InGaN 3D nano-scale dots suffer 
more additional quantum confinement than the case of embedded on InGaN 2D wetting layer.

\section{REFERENCES}

1. S. Nakamura, M. Senoh, S. Nagahama, N. Iwasa, T. Yamada, T. Matsushita, Y. Sugimoto, and H. Kiyoku, Jpn. J. Appl. Phys. Lett. 36, L1059 (1997).

2. S. Nakamura, M. Senoh, S. Nagahama, N. Iwasa, T. Yamada, T. Matsushita, H. Kiyoku, Y. Sugimoto, H. Umomoto, M. Sano, and K. Chocho, Appl. Phys. Lett. 72, 211 (1998).

3. S. Keller, B.P. Keller, M.S. Minsky, J.E. Bowers, U.K. Mishra, S.P. DeenBaars, W. Seifert, J. Cryst. Growth, 189/190, 29 (1998).

4. W. Liu, K.L. Teo, M.F. Li, S.J. Chua, K. Uchida, H. Tokunaga, N. Akutsu, K. Matsumoto, J. Cryst. Growth, 189/190, 648 (1998).

5. T. Takeuchi, S. Sota, H. Sakai, H. Amanoa, I. Akasaki, Y. Kaneko, S. Nakagawa, Y. Yamaoka, N. Yamada, J. Cryst. Growth, 189/190, 616 (1998).

6. K. Hiramatsu, Y. Kawaguchi, M. Shimizu, N. Sawaki, T. Zheleva, R.F. Davis, H. Tsuda, W. Taki, N. Kuwano, K. Oki, MRS Internet J. Nitride Semicond. Res. 2, 6 (1997).

7. J.-Y. Marzin and G. Bastard, Solid State Commun. 92, 437 (1994).

8. H. Jiang and J. Singh, Phys. Rev. B 56, 4696 (1997).

9. Y.-W. Mo, D.E. Savage, B.S. Swartzentruber, and M.G. Lagally, Phys. Rev. Lett. 65 , 1020 (1990).

10. C. Lobo and R. Leon, J. Appl. Phys. 83, 4168 (1998).

11. J.I. Pankove, Optical processes in semiconductors, (Dover publication Inc., NY. 1971) p. 120.

\section{ACKNOWLEDGEMENTS}

This work has been supported by the KOSEF and the MOST of Korea through the Semiconductor Physics Research Center at Chonbuk National University. 